\title{
A TRANS-PACIFIC COLLABORATION: DYNAMICAL EXPERIMENTS AND EXPERIENCES
}

I first met Soji Tsuchiya in 1979 at the XIV International Symposium on Free Radicals at the Kwansai Gakuin University in Sanda. At that time I had no idea what an enormous impact we would have on each other. In fact, Soji is almost completely absent from my memories of the meeting. My memories of that trip are dominated by my overwhelming first impressions of Japan, my first meeting with Soji's collaborator, Katsumi Sakurai (to whom I owned my initial scientific success as a postdoc with the late H.P. Broida, but that is another story), and one incredible post-conference day in Tokyo in which David Harris and I visited both the Hongo and Komaba campuses of the University of Tokyo and the Tokyo Institute of Technology (and gave talks at two of the three universities)! I remember discussing the Tsuchiya-Sakurai plan for "cascade type excitation" with Katsumi and realizing how lucky it was for me that our essentially identical "Stimulated Emission Pumping" experiments on $\mathrm{I}_{2}$ had already begun to work. But at that time I had absolutely no interest in polyatomic molecules, especially none in IVR or chaos, and I suspect Soji and Katsumi had little or no interest in diatomic molecules. If it were not for my collaborator at MIT, Jim Kinsey, Soji Tsuchiya and I would probably not have collided so strongly and fruitfully.

I did not discover until 8 years later what a shock the MIT SEP experiments were to Soji! In November, 1987, I wrote to Soji about his acetylene Zeeman quantum beat (ZQB) experiments [Chem. Phys. Lett. 140, 20 (1987)] in which he scooped us: "I note with great interest your paper on $\mathrm{HCCH}$ Zeeman Quantum Beast! This is a problem we at MIT and, in collaboration with the Maurice Lombardi-Remy Jost group at Grenoble, have been working on for about 5 years, but have not had the courage to write about ..." In Soji's reply he seemed to be more interested in our SEP than ZQB experiments: "The Thesis (Evan Abramson's) is extremely valuable for us since we are working on the SEP spectroscopy of vibrationally highly excited $\mathrm{SO}_{2}$. For many years, we have been spending a lot of efforts to take a SEP spectrum of a triatomic molecule. Enclosed is a preprint of our first report. I hope that you would be interested in it." Of course I was extremely interested! This launched the MIT-Tokyo collaboration.

That collaboration was one of the most important events in my career and I suspect Soji's also. Between March, 1989 and August, 1993, I made five visits to the Tsuchiya laboratory, seven MIT graduate students (George Adamson, Chris Gittins, David Jonas, Theresa Kavanaugh, Jim Lundberg, Bhavani Rajaram, and Stephani 
Solina) made 1 or 2 month research visits to Japan (six of these to the Tsuchiya labs), and six of Soji's colleagues or students (Kouichi Ichimura, Hideto Kanamori, Jun Miyawaki, Yasuhiro Ohshima, Ken Onda, and Kaoru Yamanouchi) made multimonth research visits to my laboratory. Several of these visits led to projects that have become the most important themes of my present research.

One of our most exciting but tumultuous collaborative projects was an attempt to interpret some low resolution dispersed fluorescence (DF) spectra of acetylene recorded in the Tsuchiya laboratory. This became a battle of the titans, Kaoru Yamanouchi and David Jonas. The central insight, on which we all agreed, was that "feature states" (an unresolved cluster of vibrational eigenstates) could be assigned a complete set of vibrational quantum numbers but that eigenstates, at sufficiently high $\mathrm{E}_{\mathrm{VIB}}$, were unassignable. The disagreement was over how to assign $\sim 50 \%$ of the feature states that seemed to be members of a progression built on a FranckCondon inactive mode $\left(v_{1}^{\prime \prime}\right.$, the symmetric $\mathrm{CH}$ stretch, or $v_{2}^{\prime \prime}+2 v_{5}^{\prime \prime}$, where $v_{2}^{\prime \prime}$ is the CC stretch and $v_{5}^{\prime \prime}$ is the Frank-Condon dark cis bend). For a while it seemed that the $v_{1}^{\prime \prime}$ vs. $v_{2}^{\prime \prime}+2 v_{5}^{\prime \prime}$ dispute would destroy our collaboration. David Jonas' bluntness and stubbornness stressed Kaoru Yamanouchi's courtesy and stubbornness to the breaking point. We could never have resolved this conflict without the magic of Fax and several reciprocal visits of the battling titans. Finally we figured out how to use high resolution SEP detective work to decide the matter in favour of $v_{2}^{\prime \prime}+2 v_{5}^{\prime \prime}$. The concept of assignable feature states made the combination of DF and SEP become far more powerful than either technique separately. The DF paper was finally completed in its 14th Tokyo draft! I'm sure Soji is just as proud of the final results as I am.

It has been a surprising and rewarding collaboration. I am certain that Soji Tsuchiya's "retirement" to the Japan Women's University will not put an end to this collaboration. In fact, Soji and I have begun our next joint project.

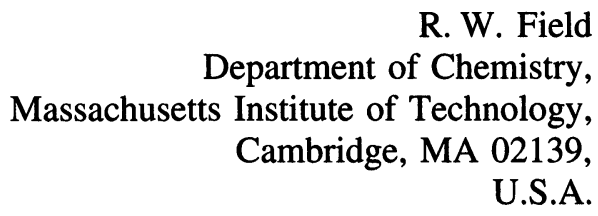

